\title{
Saúde urbana e território: dos desafios pré e durante a pandemia às respostas pós-pandemia
}

\section{Territorial and urban health: from pre-pandemic and pandemic challenges to post-pandemic responses}

\author{
Raul Borges Guimarães ${ }^{a}$ \\ (D) https://orcid.org/0000-0002-9925-5374 \\ E-mail: raul.guimaraesळunesp.br \\ Nuno Marques da Costa ${ }^{b}$ \\ (D) https://orcid.org/0000-0003-4859-9668 \\ E-mail: nunocosta®campus.ul.pt \\ Paulo Nuno Nossac \\ (D) https://orcid.org/0000-0001-5000-8754 \\ E-mail: paulonnossaðgmail.com \\ aUniversidade Estadual Paulista Júlio de Mesquita Filho. \\ Faculdade de Ciências e Tecnologia. Presidente Prudente, SP, \\ Brasil. \\ buniversidade de Lisboa. Instituto de Geografia e Ordenamento \\ do Território. Centro de Estudos Geográficos. Lisboa, Portugal. \\ 'Universidade Coimbra. Faculdade de Letras. Centro de Estudos \\ de Geografia e Ordenamento do Território. Coimbra, Portugal.
}

\section{Correspondência}

Raul Borges Guimarães

Universidade Estadual Paulista Júlio de Mesquita Filho,

Faculdade de Ciências e Tecnologia. Rua Roberto Simonsen, 305.

Presidente Prudente, SP, Brasil. CEP 19060-900.
Este dossiê foi planejado no primeiro semestre de 2019, quando não estava no horizonte a crise mundial que se estabeleceu a partir da pandemia da Covid-19. Nesse mesmo ano, a Organização Mundial da Saúde (OMS) havia publicado uma lista de revisão do que considerava ser as principais dez ameaças globais; dentre elas: os efeitos da poluição relacionados com as alterações climáticas; as pandemias decorrentes dos vírus influenza; a expansão do ebola e outros agentes patogênicos de alto risco; a resistência aos antibióticos; a dengue; e a resistência à vacinação (WHO, 2019).

De fato, dentre as ameaças identificadas pela OMS, o risco de uma pandemia se efetivou, não dos vírus influenza, mas de um o novo coronavírus denominado Sars-Cov-2. Ele tem gerado o desafio de garantir acesso aos serviços de saúde para todos pacientes com deficiência respiratória aguda, principalmente nos leitos de unidade de tratamento intensivo equipados com respiradores/ ventiladores, equipamentos de alta complexidade médica (CORONAVIRUS..., 2020).

Certamente os temas abordados nos textos reunidos neste dossiê se tornarão ainda mais relevantes nos próximos anos, por decorrência do impacto causado pela pandemia da Covid-19, uma vez que envolvem evidências teóricas e empíricas que colocam a saúde no centro de problemáticas relevantes para as sociedades contemporâneas, destacando-se os espaços urbanos, onde reside a maioria da população.

Nas áreas urbanas, alguns destes problemas ganham importância adicional, nomeadamente aqueles que são mais impactados pelas alterações climáticas, tais como: afetação de níveis de poluição do ar, ciclos patogênicos mais curtos, alteração na disponibilidade e no controle da qualidade da 
água. A estes problemas somam-se questões de segurança alimentar, que perante a massificação do consumo e as necessidades de manter uma produção sem falhas, transformam os alimentos em canais portadores de agrotóxicos, de descontaminantes e de conservantes. Estes acontecimentos reforçam a lista de riscos, em especial os problemas respiratórios, cardiovasculares e do sistema imunológico.

O relatório de 2016 da OMS, intitulado Global report on urban health: equitable, healthier cities for sustainable development, identifica algumas dimensões estruturantes: (1) a redução das desigualdades em saúde e respetiva promoção de sistemas universais de saúde em todo o mundo; (2) o combate às doenças transmissíveis e o controle da expansão das não transmissíveis; e, ainda, (3) diminuir a fome e a desnutrição. Para atingir estes objetivos o planeamento urbano deve, segundo o relatório, garantir saneamento e água potável para todos, desenvolver um desenho urbano que promova uma cidade mais saudável e inclusiva, melhorar a mobilidade urbana, aumentar as condições da habitação e garantir a segurança na cidade (WHO, 2016).

As orientações da OMS cruzam com os Objetivos do Desenvolvimento Sustentável (ODS) da Organização das Nações Unidas para muito além do “Objetivo 3-Saúde e Bem-Estar". Ela se liga de forma transversal aos restantes ODS, nos quais a proteção dos recursos, a racionalização dos consumos e o combate às desigualdades têm efeitos em todos os domínios da saúde (17 OBJETIVOS..., 2015).

É neste contexto, que o presente dossiê, através dos seus seis artigos, traz para a discussão a importância da transversalidade da saúde no território, centrada nos seguintes temas:

- o processo de envelhecimento das sociedades e os desafios inerentes a ele;

- a questão da equidade no acesso à saúde; e

- a importância do planejamento para desenvolver respostas no sentido de construir cidades mais saudáveis.

Tomando o primeiro tema, sabemos que o processo de envelhecimento demográfico acarreta novos desafios ao território, nomeadamente no acesso à saúde. Porém por via do planejamento, da gestão ambiental e da intervenção urbana, se podem encontrar soluções que reforçam as complexas conexões da saúde com o espaço em constante transformação e movimento.

O forte crescimento da população urbana com idade igual ou superior a 65 anos tem gerado intenso debate, nos contextos acadêmico e político: questiona-se se a cidade, ou as áreas metropolitanas em geral, são territórios aptos para potencializarem o bem-estar de seus residentes. A Organização para a Cooperação e Desenvolvimento Econômico (OCDE) perspetiva para os países membros que, até 2050, ocorra um aumento de $25,1 \%$ de idosos a residir em áreas urbanas, sendo que, no presente, $43,2 \%$ dos habitantes com 65 ou mais anos já vivem em cidades (OECD, 2015, p. 5). Van Hoof et al. (2018, p. 2, tradução nossa) retomam a discussão sobre o potencial de bem-estar dos idosos no espaço urbano admitindo que, em determinadas circunstâncias: "Envelhecer no lugar (i.e., viver na comunidade, com algum nível de independência e não em cuidado residencial), é muitas vezes visto como um ideal".

Em Portugal, o envelhecimento populacional foi particularmente acentuado ao longo de toda a década de 1990, tendo ultrapassado o número de 100 idosos para cada 100 jovens na transição do século (2001). Uma trajetória semelhante está prevista para o Brasil, ainda que com algum deferimento. 0 país apresenta hoje um índice de envelhecimento idêntico ao que era registado em Portugal na segunda metade dos anos 1980, sendo que as estimativas da OMS apontavam 2037 como o ano em que o número de idosos ultrapassaria o da população jovem (WHO, 2010). Estima-se que, em 2025, o Brasil seja o quinto país do mundo com maior número absoluto de idosos (33,4 milhões) (OPAS, 2005, p. 10). De acordo com o relatório Growing old in an older Brazil, publicado pelo Banco Mundial:

A população idosa aumentará de cerca de $11 \%$ do total da população em idade ativa, em 2005, para $49 \%$ até 2050 , enquanto a população em idade escolar diminuirá de cerca de $50 \%$ para $29 \%$ da população em idade ativa em 2050. (Gragnolati et al., 2011, p. XXVI) 
$\mathrm{O}$ artigo apresentado por Paulo Nossa neste dossiê, intitulado "Envelhecimento, financiamento e inovação nos sistemas de saúde: uma discussão necessária para a manutenção do direito à saúde”, enfatiza a importância de se promover atuações concertadas e transversais no domínio da saúde. Para que os quadros de atuação ganhem eficiência e eficácia, importa alcançar formas inovadoras na gestão e promoção da saúde, reintroduzindo o lugar como uma categoria fundamental nas políticas públicas de saúde (Nossa, 2017).

A questão do envelhecimento e a sua relação com os vários determinantes de saúde é igualmente abordada neste dossiê no artigo apresentado por C. M. Oliveira, S. M. Alves, H. Teixeira, T. Economou, J. Pereira-da-Silva, T. Bailey e M. F. Pina, intitulado "Efeitos regionais da composição da água potável no risco de fratura do quadril. Uma análise espacial das admissões do hospital nacional de 2000 a 2010 em Portugal”. Este texto enfatiza a relação ambiental com a saúde dos idosos. Dependendo da dose de alguns componentes da água, como alumínio ou cádmio, assiste-se ao deteriorar da saúde óssea, enquanto outros, como cálcio, magnésio e manganês, parecem proteger essa mesma saúde. Os autores mostram que a deposição de tais minerais e metais nos ossos (mesmo em doses baixas), como resultado da exposição a longo prazo, pode contribuir para a sua fragilidade (por exemplo, alumínio) ou para a sua resistência (por exemplo, magnésio e cálcio).

A problemática do envelhecimento pode ainda ser abordada sob o ponto de vista da equidade em saúde, na vertente do acesso aos seus serviços. Neste contexto, para além das barreiras físicas existentes no acesso aos cuidados de saúde e que tradicionalmente são abordadas na literatura (Ensor; Cooper, 2004; Furtado; Pereira, 2010), frequentemente relacionadas com o progressivo declínio funcional dos mais idosos, deve-se também discutir a conceitualização e a diversidade das barreiras de acesso, algumas das quais associadas ao planejamento e desenho da própria cidade, mesmo que, intencionalmente, não tentem condicionar o acesso das populações. De acordo com a OMS (WHO, 2015, p. 91), as barreiras de acesso aos cuidados de saúde dos idosos são diversas e podem passar por dificuldades de transporte; impossibilidade financeira, mesmo em países de elevado rendimento; ou, simplesmente, resultam de uma abordagem pouco empática, ampliada por preconceito e uma comunicação deficiente, pressupondo os relatos dos mais idosos como insuficientes ou pouco credíveis para que possam ser valorizados como doença, desencadeando nestes utilizadores a perceção de mau trato ou mesmo de indesejabilidade. Este tipo de apreciação reforça a ideia de que mantivemos, por muito tempo, o status quo das políticas e práticas em saúde que são moldadas por um paradigma de atuação paternalista, autoritário e curativo, reformando de modo insuficiente o modus operandi dos cuidados de saúde, particularmente ao nível dos cuidados primários, pouco centrados nas alterações das necessidades evidenciadas pelo perfil demográfico das comunidades, com escassos recursos alocados à promoção e prevenção da saúde. Estes fatos são consequência de um modelo de gestão centralizado e hierárquico (top-down) e que, em alguns países, permaneceu excessivamente centrado na doença e no cuidado hospitalar, em que as necessidades específicas de alguns grupos, entre os quais os mais idosos, podem receber atenção insuficiente (Nossa, 2017).

Esta abordagem continua pertinente na literatura por via das insuficiências detectadas neste público específico. Baer et al. (2016) enfatizam esta problemática sublinhando que, em diversas partes do mundo, o direito do acesso dos idosos aos cuidados de saúde ainda é uma questão de direitos humanos, que não pode e não deve ser encarada de modo progressivo, requerendo atenção imediata e holística por parte dos Estados. No que respeita à acessibilidade, além de os serviços estarem fisicamente disponíveis para os mais idosos, é importante que os programas e as ferramentas de saúde estejam adaptados às competências e às necessidades dos idosos, incluindo patamares considerados básicos nas sociedades abastadas:

Por exemplo, a lei internacional dos direitos humanos, explicitamente, solicita a disponibilidade de medicamentos essenciais como parte das obrigações mínimas essenciais ao direito à saúde. A evidência sugere também que os esforços para avançar em direção à cobertura universal dos 
dispositivos de assistência necessários e aos medicamentos essenciais têm grande potencial para melhorar a saúde e a independência dos idosos. (Baer et al., 2016, p. S209, tradução nossa)

No meio urbano a situação não se altera, tanto mais que a cidade é crescentemente ocupada por idosos, cujo acesso aos serviços de saúde e aos níveis de diferenciação é necessário debater e acautelar. Essa proposta está presente no texto de E. Marques da Costa, N. Marques da Costa, A. Louro e M. Barata, "Geografias" do acesso dos idosos aos cuidados de saúde primários na Área Metropolitana de Lisboa, Portugal - um território de diferenças". Embora seja consensual que o conceito de acesso está para além da acessibilidade física, os autores revisitam com detalhe os conceitos de acesso e de acessibilidade nas suas múltiplas componentes, almejando a sua adaptação a um público-alvo idoso (mais de 65 anos). Pelo expresso, o artigo em pauta publicado neste dossiê é relevante, pois avalia, a partir da modelação da rede viária em SIG da Área Metropolitana de Lisboa, Portugal, o potencial de cobertura populacional considerando a acessibilidade física da população idosos aos equipamentos de cuidados de saúde primários. Tem-se em conta diferentes limiares de distância-tempo, cruzando com informação obtida através de inquérito esclarecendo questões específicas de acesso e procura à escala da freguesia, levantando tópicos relacionadas a rendimento, acesso a automóvel privado e variação da localização de equipamentos de saúde em função da densidade populacional.

Ainda no registo da equidade no acesso à saúde, podemos discutir uma das 10 ameaças da OMS (WHO, 2019), relacionada com a expansão de agentes patogênicos de alto risco. Recentemente, no contexto de atuação à difusão da pandemia gerada pelo SarsCoV-2, Wang, Ng e Brook (2020) sublinharam que cenários de contenção bem-sucedidos, como o da Tailândia, dependem de contextos de saúde pública bem treinados e que integram estruturas de gestão multinível, processos de comunicação culturalmente adaptados e instrumentos de atuação flexíveis adaptados às necessidades de cada lugar.

Os autores P. Peiter, R. Pereira e I. França, no artigo “Análise de dimensões do acesso à saúde das crianças com Síndrome Congênita de Zika (SCZ) na Região Metropolitana do Rio de Janeiro" (RMRJ), procuram analisar a disponibilidade e acessibilidade dos serviços de saúde para o tratamento e reabilitação da SCZ na RMRJ, mostrando as diferenças entre os vários lugares, mais exatamente entre os vários municípios. 0 estudo mostra que os mais afetados, entre 2015 e 2017, foram o Rio de Janeiro, com 127 casos; seguido por Duque de Caxias, com 16; e Nova Iguaçu, com oito casos. Contudo foi no Rio de Janeiro, também, que se verificou melhor acesso aos centros especializados em reabilitação, em contraponto à área leste da RMRJ II, que registrou as piores condições de acesso. Os autores referem ainda que o acesso aos serviços também varia conforme as condições dos usuários, no caso, os familiares (primordialmente mães) de crianças afetadas pela SCZ.

O último tema do dossiê relaciona-se com o planejamento de cidades saudáveis. O projeto Cidades Saudáveis congrega algumas destas premissas, ao enfatizar a importância prática de uma gestão holística da cidade e da sua responsabilidade na prossecução das condições de saúde e de inclusão. Ele sublinha a importância da ação local e das ligações que a gestão e promoção da saúde detêm como o planeamento urbano, o zoneamento, a construção/ adequação de espaços verdes, a habitação, os transportes, entre outros (Louro; Marques da Costa; Marques da Costa, 2017). Desde a década de 1980, vários movimentos urbanísticos produziram uma abordagem crítica e apontaram a necessidade de se desenharem cidades sustentáveis, capazes de combaterem a expansão urbana dominante. Esse alastramento urbano, também conhecido como urban sprawl, é marcada por grandes impactos ambientais associados ao crescimento horizontal da cidade, que gera periferias economicamente segregadas; e à utilização crescente do automóvel como forma privilegiada de deslocação, que agrava modos de vida progressivamente mais sedentários, com evidentes prejuízos para a saúde (Marques da Costa, 2016).

Como sublinha Tsouros (200o), o contexto vigente de cidade saudável é, em si próprio, um processo aspiracional, dinâmico e inacabado, uma vez que uma cidade saudável não é aquela que alcançou um nível de saúde específico, mas sim 
a cidade que, consciente do seu nível de saúde, desenvolve esforços para o melhorar de modo contínuo. Takano (2003, p. 6, tradução nossa) reforça esta perspetiva, enfatizando as vantagens de uma integração de políticas responsabilizadoras desta ação à escala local:

O princípio das Cidades Saudáveis está sendo desenvolvido como um pilar fundamental para o avanço da saúde dos habitantes das cidades, principalmente em escala municipal, assegurando a incorporação dos aspetos relacionados à saúde em todas as políticas e obras da cidade, incluindo os níveis de desenvolvimento, planejamento e gestão urbana; i.e., como uma estrutura municipal para apoiar a saúde dos munícipes.

Materializando a dimensão da resposta das cidades, F. A. Lima e S. C. Lima, no texto "Construindo cidades saudáveis: a instrumentalização de políticas públicas intersetoriais de saúde a partir do Planejamento Estratégico Situacional”, sublinham a pertinência de refletirmos sobre o contexto histórico evolutivo associado ao planejamento integrado da cidade, ao modo como ela evoluiu, procurando corrigir, ciclicamente, contexto divergentes e excludentes do ponto de vista social e sanitário, com incontornáveis reflexos na saúde das populações. Tal como sublinham os autores, este é um processo dinâmico e continuado que não deve descartar a construção histórica e social do espaço urbano, evitando tentações higienistas, sejam estas de natureza social, sanitária ou educacional.

Complementado este registo, Hélio Hirao retrata o caminho a percorrer, "Da cidade dos afetos para a cidade saudável”, sublinhando e debatendo a associação entre o ambiente construído, na sua dimensão social e material, e a apropriação que se faz dos territórios associados às múltiplas e complexas particularidades da saúde num espaço em transformação e movimento. No contexto das cidades, as quais o autor admite serem "cada vez mais híbridas e desterritorializadas", argumenta-se a necessidade de nos munirmos de outras perspetivas que nos ajudem a interpretar as suas singularidades socioculturais e espaciais, através de uma leitura alternativa de ações de intervenção nos corpos (homem, arquitetura e território). 0 texto nos propõe um singular exercício de reflexão e observação, tendo como objeto de trabalho os vazios urbanos (terrain vague) presentes em áreas desvalorizadas do ponto de vista imobiliário. Através de múltiplas interposições propostas para ressignificar micro espaços de insurgências dos moradores nos lotes vagos, algumas vezes com um aproveitamento cênico e plástico improvável, complementada por uma geografia sonora, o autor conjuga diversos exemplos de cartografia de intervenção. Por meio desses exemplos, procura captar experiências subjetivas de forças e de afetos, propiciando uma leitura alternativa, fortalecedora de relações de bemestar físico, mental e social e que, por isto, promove uma cidade saudável.

O contexto teórico aqui apresentado, bem como os dados empíricos discutidos em alguns artigos, pretendem contribuir para fortalecer um debate plural e multidisciplinar em torno da problemática: saúde urbana e território. Como fica demonstrado e foi solicitado pela OMS (2010) na última década, é importante que a questão da saúde seja transversal a todas as políticas; que se debata uma realidade multiescalar, que envolva responsabilidade, transparência e participação. É fundamental, também, que essas políticas sejam inclusivas e que se adaptem às especificidades dos diferentes grupos, no caso em pauta, os mais idosos, que necessitam dos serviços públicos uma atuação que garanta acesso aos cuidados e que adapte as cidades às diferentes necessidades de seus habitantes.

\section{Referências}

17 OBJETIVOS para transformar nosso mundo. ONU Brasil, Brasília, DF, 2015. Disponível em: <https://nacoesunidas.org/pos2015/>. Acesso em: 22 mar. 2020.

BAER, B. et al. The right to health of older people. Gerontologist, Oxford, v. 56, p. S206-S217, 2016. Suplemento 2. Disponível em:

<https://bit.ly/2AhwykJ>. Acesso em: 2 mar. 2020.

CORONAVIRUS disease (COVID-19) pandemic. World Health Organization, Genebra, 2020. Disponível em: <https://bit.ly/2ZK2C50>.

Acesso em: 5 maio 2020. 
ENSOR, T.; COOPER, S. Overcoming barriers to health service access and influencing the demand side through purchasing. Washington, DC: World Bank's Human Development Network, 2004. (Working Paper, 31598). Disponível em: <https://bit.ly/36Mf7Fv>. Acesso em: 2 set. 2012.

FURTADO, C.; PEREIRA, J. Equidade e acesso aos cuidados de saúde. Lisboa: Universidade Nova de Lisboa, 2010. Documento de trabalho. Disponível em: <https://bit.ly/3gu2mDJ>. Acesso em: 2 set. 2012.

GRAGNOLATI, M. et al. Growing old in an older Brazil: implications of population aging on growth, poverty, public finance and service delivery. Washington, DC: World Bank, 2011. Disponível em: <https://bit.ly/2Xy5Psg>. Acesso em: 10 out. 2013.

LOURO, A.; MARQUES DA COSTA, E.; MARQUES DA COSTA, N. A cidade saudável no planeamento municipal: exemplos de atuação na Área Metropolitana de Lisboa. In: SOARES, B. R. et al. (Org.). Construindo cidades saudáveis: utopias e práticas. Uberlândia: Assis, 2017. p. 33-68.

MARQUES DA COSTA, E. Da ação em saúde à ação para a saúde. In: ALDEMIR, J.; ALVES, G. (Ed.). Geografia da saúde: ambientes e sujeitos sociais no mundo globalizado. Manaus: Universidade Federal da Amazónia, 2016. p. 71-93.

NOSSA, P. Participação, governação e inclusão: os contributos da rede de cidades saudáveis no domínio da saúde e do trabalho. In: SOARES, B. R. et al. (Org.). Construindo cidades saudáveis: utopias e práticas. Uberlândia: Assis, 2017. p. 123-16o.

OECD - ORGANISATION FOR ECONOMIC COOPERATION AND DEVELOPMENT. Ageing in cities: policy highlights. Paris, 2015. Disponível em: <https://bit.ly/3c9aPca>. Acesso em: 2 dez. 2019.

OMS - ORGANIZAÇÃO MUNDIAL DA SAÚDE. Declaração de Adelaide sobre a saúde em todas as políticas: no caminho de uma governança compartilhada, em prol da saúde e do bem-estar. Genebra, 2010. Disponível em: <https://bit.ly/2TKBNAw>. Acesso em: 5 maio 2016. OPAS - ORGANIZAÇÃO PAN-AMERICANA DA SAÚDE. Envelhecimento ativo: uma política de saúde. Brasília, DF, 2005. Disponível em: <https://bit.ly/2MgirsI >. Acesso em: 2 jan. 2020.

TAKANO, T. Healthy cities \& urban policy research. Londres: Spon Press, 2003.

TSOUROS, A. (Ed.). World Health Organization Healthy Cities Project: a project becomes a movement: review of progress 1987 to 1990. Milão: WHO Regional Office for Europe, 2000. Disponível em: <https://bit.ly/2zumoxs >. Acesso em: 12 dez. 2002.

VAN HOOF, J. et al. The challenges of urban ageing: making cities age-friendly in Europe.

International Journal of Environmental Research and Public Health, Basel, v. 15, n. 11, p. 2473, 2018. Disponível em: <https://bit.ly/3gpRıVr>. Acesso em: 20 mar. 2020.

WANG, C. J.; NG, C.Y.; BROOK, R. H. Response to COVID-19 in Taiwan: Big Data Analytics, New Technology, and Proactive Testing. JAMA, Chicago, 3 Mar. 2020. Disponível em: <https://bit.ly/2yB3CDU>. Acesso em: 20 mar. 2020. WHO - WORLD HEALTH ORGANIZATION. World population prospects: the 2010 revision. Genebra, 2010. Disponível em: <https://bit.ly/3dcbx9K>. Acesso em: 1 jul. 2012.

WHO - WORLD HEALTH ORGANIZATION. World report on ageing and health. Genebra, 2015. Disponível em: <https://bit.ly/3gDNvqB>. Acesso em: 1 jun. 2020.

WHO - WORLD HEALTH ORGANIZATION. Global report on urban health: equitable, healthier cities for sustainable development. Genebra, 2016.

Disponível em: <https://bit.ly/36Bdjid>.

Acesso em: 2 fev. 2020.

WHO - WORLD HEALTH ORGANIZATION. Ten threats to global health in 2019. Genebra, 2019. Disponível em: <https://bit.ly/2zslWQj>. Acesso em: 22 mar. 2020.

Recebido: 06/05/2020

Aprovado: 13/05/2020 